\title{
Surrogate-driven deformable motion model for organ motion tracking in particle radiation therapy
}

\author{
Aurora Fassi ${ }^{1}$, Matteo Seregni ${ }^{1}$, Marco Riboldi ${ }^{1,2}$, \\ Pietro Cerveri ${ }^{1,2}$, David Sarrut ${ }^{3,4}$, Giovanni Battista Ivaldi ${ }^{5}$, \\ Paola Tabarelli de Fatis ${ }^{6}$, Marco Liotta ${ }^{6}$ and Guido Baroni ${ }^{1,2}$ \\ 1 Dipartimento di Elettronica, Informazione e Bioingegneria, Politecnico di Milano, P.zza Leonardo \\ da Vinci 32, I-20133 Milano, Italy \\ 2 Bioengineering Unit, Centro Nazionale di Adroterapia Oncologica, Strada privata Campeggi, \\ I-27100 Pavia, Italy \\ 3 Department of Radiotherapy, Centre Léon Bérard, 28 Promenade Léa et Napoléon Bullukian, \\ F-69008 Lyon, France \\ ${ }^{4}$ CREATIS, CNRS UMR 5220, INSERM U1044, Université Lyon 1, INSA-Lyon, F-69621 \\ Villeurbanne, France \\ 5 Department of Radiation Oncology, Fondazione Salvatore Maugeri, Via Salvatore Maugeri 8-10, \\ I-27100 Pavia, Italy \\ ${ }^{6}$ Department of Medical Physics, Fondazione Salvatore Maugeri, Via Salvatore Maugeri 8-10, \\ I-27100 Pavia, Italy \\ E-mail: aurora.fassi@polimi.it
}

Received 5 September 2014, revised 17 December 2014

Accepted for publication 19 December 2014

Published 23 January 2015

\section{Abstract}

The aim of this study is the development and experimental testing of a tumor tracking method for particle radiation therapy, providing the daily respiratory dynamics of the patient's thoraco-abdominal anatomy as a function of an external surface surrogate combined with an a priori motion model. The proposed tracking approach is based on a patient-specific breathing motion model, estimated from the four-dimensional (4D) planning computed tomography (CT) through deformable image registration. The model is adapted to the interfraction baseline variations in the patient's anatomical configuration. The driving amplitude and phase parameters are obtained intrafractionally from a respiratory surrogate signal derived from the external surface displacement. The developed technique was assessed on a dataset of seven lung cancer patients, who underwent two repeated 4D CT scans. The first 4D CT was used to build the respiratory motion model, which was tested on the second scan. The geometric accuracy in localizing lung lesions, mediated over all breathing phases, ranged between 0.6 and $1.7 \mathrm{~mm}$ 
across all patients. Errors in tracking the surrounding organs at risk, such as lungs, trachea and esophagus, were lower than $1.3 \mathrm{~mm}$ on average. The median absolute variation in water equivalent path length (WEL) within the target volume did not exceed $1.9 \mathrm{~mm}$-WEL for simulated particle beams. A significant improvement was achieved compared with error compensation based on standard rigid alignment. The present work can be regarded as a feasibility study for the potential extension of tumor tracking techniques in particle treatments. Differently from current tracking methods applied in conventional radiotherapy, the proposed approach allows for the dynamic localization of all anatomical structures scanned in the planning CT, thus providing complete information on density and WEL variations required for particle beam range adaptation.

Keywords: tumor tracking, particle therapy, 4D CT motion model, external surface surrogate

\section{Introduction}

Organs in the thoracic and abdominal regions can significantly move as a result of physi-ological processes, mainly due to respiration (Keall et al 2006). Interfraction and intrafraction organ motion may seriously affect the accuracy and effectiveness of radiation dose delivery, by inducing geometric uncertainties in the localization of target and organs at risk (OARs). In highly selective treatments, such as those delivered by means of charged particle beams, organ motion management is strictly demanded (Bert and Durante 2011). The potentially most effi-cient motion-compensated strategy is tumor tracking, which allows for continuous irradiation in free-breathing conditions (Riboldi et al 2012). The application of tumor tracking techniques requires the real-time localization of the lesion for the dynamic steering and conformation of the radiation beam (Keall et al 2001, Schweikard et al 2004). Target position can be directly estimated through in-room x-ray imaging systems, such as digital radiography or fluoroscopy (Shirato et al 2000). In case of low-contrast lesions, radiopaque clips are usually implanted near the tumor to facilitate its detection in $\mathrm{x}$-ray projections. Tracking methods based on radiographic imaging provide high accuracy in tumor localization (Poulsen et al 2010), but involve the invasive use of ionizing radiation. An alternative approach for direct tumor tracking relies on implanted transponders, which are detected continuously by means of external electromagnetic receivers (Balter et al 2005, Keall et al 2014). However, the implantation of internal markers, like radiopaque clips or electromagnetic transponders, is a widely debated issue especially for lung tumors, due to the possible risk of pneumothorax or clip migration (Imura et al 2005). Moreover, considering particle therapy, implanted devices may alter dose distribution in the target volume (Bert and Durante 2011).

Tumor motion can also be indirectly inferred from an external respiratory surrogate, typi-cally represented by the displacement of the patient's thoraco-abdominal surface (Keall et al 2006). Non-invasive optical tracking devices can be used to acquire surface motion, by means of opto-electronic localizers that reconstruct the trajectory of passive markers placed on the patient's skin (Baroni et al 2007) or through surface imaging systems that scan the entire markerless surface (Bert et al 2005). The non-invasivity of this approach allows for continuous monitoring of intrafraction motion during the whole treatment course. However, 
the correlation between the external surrogate and the internal tumor position needs to be fre-quently verified to avoid residual errors (Seregni et al 2012). In the current tracking systems applied in conventional photon radiotherapy, external-internal correlation models are initial-ized before treatment and periodically updated during the whole fraction by acquiring surface motion simultaneously with x-ray projections (Schweikard et al 2004, Hoogeman et al 2009, Takayama et al 2009, Kilby et al 2010, Depuydt et al 2013).

Charged particle therapy with active beam scanning offers excellent conditions for real-time motion compensation through tumor tracking techniques, due to the possibility of fast beam steering and depth-range adaptation (Schardt et al 2010, Riboldi et al 2012). However, active scanning systems are more sensitive to organ motion as a result of the interplay between the moving tumor and the scanned beam (Bert and Durante 2011). Moreover, the motion of organs within the beam path can change the radiological water equivalent path length (WEL), thus altering the distribution of the delivered dose (Mori et al 2007, 2008). Although the technical feasibility of a tracking system with scanned beams has already been demonstrated (Saito et al 2009), no clinical application has been reported up to date. Current tracking meth-ods used in conventional radiotherapy (Kilby et al 2010, Keall et al 2014) cannot be directly applied to particle therapy, since they only provide the position of single internal points, such as the target. The tracking of all anatomical structures traversed by particles is instead required for obtaining tissue density variation along the beam, thus allowing for particle range adapta-tion according to the associated path length fluctuations.

Patient-specific respiratory motion models derived from four-dimensional (4D) planning computed tomography (CT) or cone beam (CBCT) have been recently investigated for tumor tracking applications (McClelland et al 2011, Fayad et al 2012, Martin et al 2013, Fassi et al 2014). These models describe the respiration-induced dynamics of the patient's inter-nal anatomy as a function of an external surface surrogate. In a previous study (Fassi et al 2014), we presented a surrogate-driven motion model obtained from the 4D planning CT through deformable image registration (DIR). Differently from other modeling approaches (McClelland et al 2011, Fayad et al 2012, Martin et al 2013), we introduced specific strategies to account for interfraction and intrafraction variations in patient's anatomical configuration and respiratory motion parameters. In the previous work (Fassi et al 2014), the developed approach was limited to the tracking of the tumoral target, restricting the motion model to a volume of interest (VOI) nearby the lesion. The aim of the present study was to investigate the potential application of the proposed modeling technique for tumor tracking in particle radia-tion therapy, extending the motion model to the entire CT volume in order to track all struc-tures of interest in the scanned patient's anatomy. The experimental tests were mainly aimed at the quantification of the geometric accuracy in estimating the position, shape and volume of lung tumors and surrounding OARs, as well as the radiological WEL variation necessary to adapt the penetration range in charged particle irradiation. This study could provide the first step towards adopting patient-specific respiratory motion models for guiding particle therapy.

\section{Methods}

\subsection{Respiratory motion model}

The methodological approach for the construction of the patient-specific respiratory motion model, already introduced in previous works (Vandemeulebroucke et al 2009, Fassi et al 2014), is summarized in the following paragraph (figure 1). The model is estimated from the 4D planning CT through DIR, obtaining a set of input deformation vector fields (DVFs) that describe the motion of each voxel between a reference volume and each frame of the 4D CT 


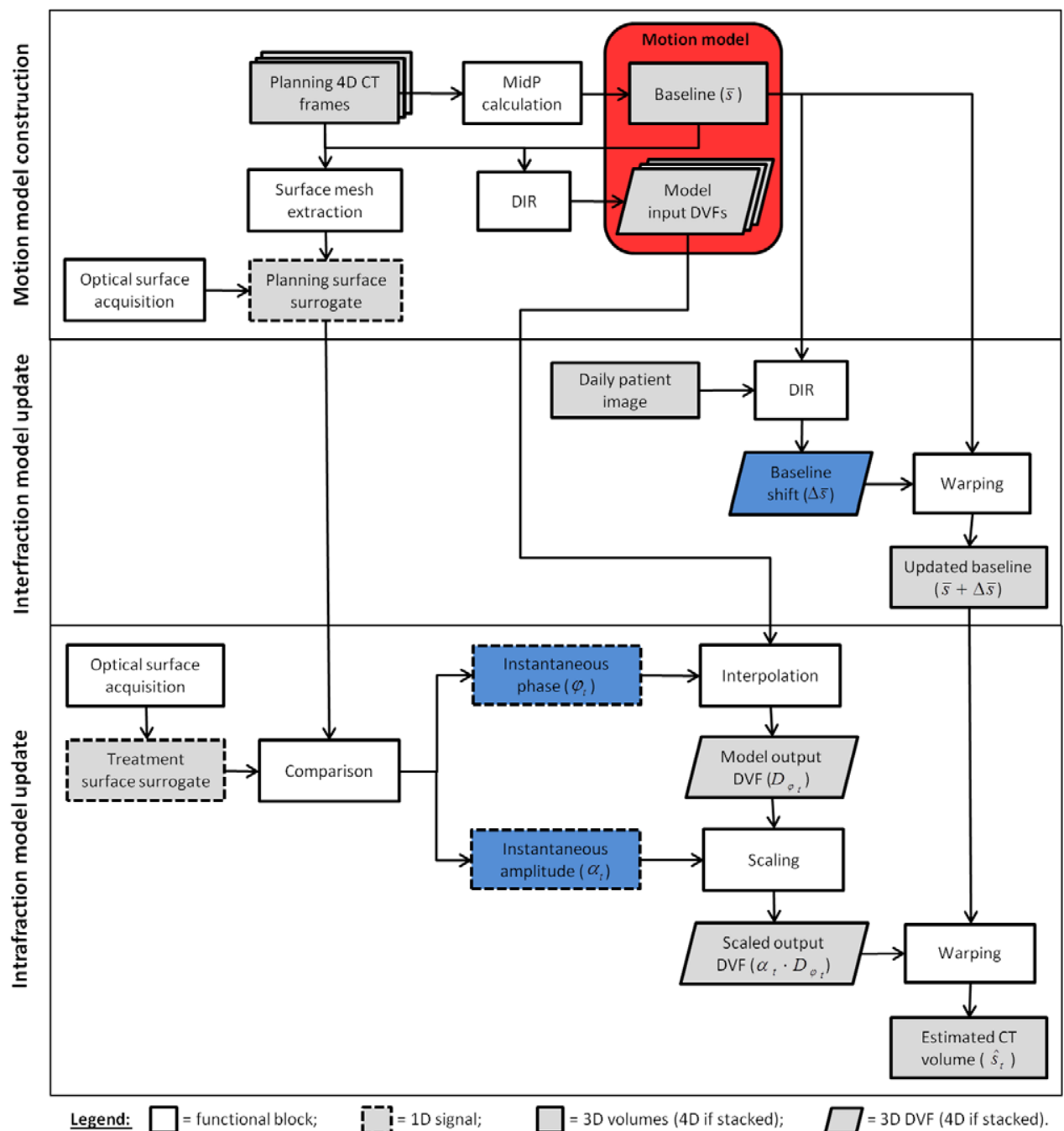

Figure 1. General workflow of the proposed tumor tracking method, including the motion model construction and update steps. The three breathing parameters of the motion model are outlined in blue.

scan. The considered reference volume is the midposition (MidP) CT, which represents the time-averaged position of the patient's anatomy during the 4D CT breathing cycle. The MidP CT is reconstructed from the 4D CT image set, as described by Wolthaus et al (2008). A multiresolution B-spline DIR algorithm (Shackleford et al 2010) is applied to register the MidP CT with each single 4D CT frame. Four DIR stages with decreasing input volume sub-sampling and B-spline grid spacing are used. A second derivative regularization term is considered to increase the smoothness of the registration.

At each temporal instant $t$, the respiratory model provides a specific motion state $\hat{s}_{t}$ as a function of three breathing parameters (baseline shift $\Delta \bar{s}$, phase $\phi_{t}$ and amplitude $\alpha_{t}$ ) according to the following equation:

$$
\hat{s}_{\mathrm{t}}=\bar{s}+\Delta \bar{s}+\alpha_{\mathrm{t}} \cdot D_{\phi_{\mathrm{t}}} .
$$




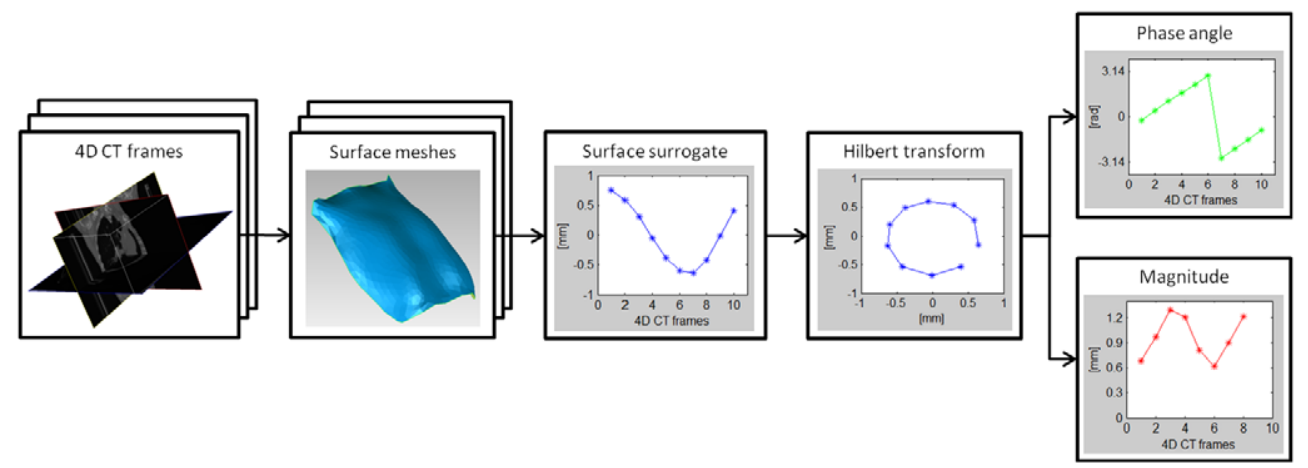

Figure 2. Illustration of the steps for the estimation of the Hilbert transform parameters from the surface meshes extracted from 4D CT frames.

The variables included in the equation are explained below and depicted in figure 1.

- The baseline $\bar{s}$ represents the reference state used for the estimation of the model input DVFs, i.e. the MidP CT.

- The baseline shift $\Delta \bar{s}$ consists in the interfraction variations of the baseline parameter and is computed as the DVF between the MidP CT and the daily patient image, e.g. the CBCT volume acquired during in-room patient setup.

- The updated baseline $\bar{s}+\Delta \bar{s}$ is obtained by warping the baseline with the baseline shift.

The phase and amplitude parameters are estimated at each time instant during irradiation by comparing the breathing surrogate signal derived from patient's surface motion during treatment with the surrogate obtained during planning CT. Surface motion can be acquired by means of optical tracking system or by segmenting the thoraco-abdominal surface mesh from the CT frames (figure 2). The Hilbert transform (Gabor 1946) applied to both treatment and planning surrogate signals is used to compute the instantaneous phase and amplitude values.

- The phase $\phi_{t}$ indicates the angular position of the desired motion state within the 4D CT breathing cycle and is obtained as the Hilbert transform phase angle (figure 2) expressed as percentage position between the 4D CT frames.

- The amplitude $\alpha_{t}$ corresponds to a scaling factor relatively to the motion measured in the 4D CT scan and is derived as the ratio between the Hilbert transform magnitude (figure 2) of the treatment surrogate and of the planning surrogate at the corresponding phase $\phi_{t}$.

- The term $D_{\phi_{t}}$ represents the model output DVF obtained by interpolating the model input DVFs in correspondence of phase $\phi_{t}$ by using cubic B-splines. The model output DVF, which is equivalent to the displacement between the motion state at phase $\phi_{t}$ and the baseline motion state, is scaled according to the amplitude factor $\alpha_{t}$, in order to take into account intrafraction variations in motion magnitude between daily and planning conditions.

- The CT volume $\hat{s}_{t}$ associated to the desired motion state is estimated by warping the updated baseline with the scaled output DVF.

\subsection{Experimental protocol}

The accuracy of the proposed tracking method was tested on seven non-small cell lung can-cer patients treated with stereotactic body radiation therapy. Each patient underwent two 
Table 1. Summary of lung patient data considered for this study. Tumor volume was computed from the end-exhale frame of the first 4D CT scan, while the range of motion was obtained as the peak-to-peak amplitude of the 3D tumor centroid trajectory. The time interval between the two 4D CT scans performed by each patient is also reported in the table.

\begin{tabular}{llcll}
\hline Patient & Tumor location & $\begin{array}{l}\text { Tumor } \\
\text { volume }\left(\mathrm{cm}^{3}\right)\end{array}$ & $\begin{array}{l}\text { Tumor range of } \\
\text { motion }(\mathrm{mm})\end{array}$ & $\begin{array}{l}\text { 4D CT time } \\
\text { interval }\end{array}$ \\
\hline P1 & Right middle lobe & 1.8 & 12.4 & Same day \\
P2 & Right upper lobe & 14.0 & 1.2 & Same day \\
P3 & Left upper lobe & 2.7 & 7.4 & Same day \\
P4 & Left upper lobe & 3.5 & 5.0 & $1 \mathrm{~d}$ \\
P5 & Left upper lobe & 7.4 & 7.1 & $7 \mathrm{~d}$ \\
P6 & Right lower lobe & 1.5 & 5.2 & $8 \mathrm{~d}$ \\
P7 & Left lower lobe & 18.4 & 3.2 & $18 \mathrm{~d}$ \\
\hline
\end{tabular}

4D CT scans within the same day (patients P1-P3) or in different days (patients P4-P7), as reported in table 1 . This allows testing the ability of the developed approach in compensating both for intrafraction (patients P1-P3) and interfraction (patients P4-P7) variations in respiratory motion parameters. Patients P1-P4 were scanned with the Philips Brilliance Big Bore CT scanner (Philips Medical Solution, Cleveland, $\mathrm{OH}$ ) at the Centre Léon Bérard in Lyon, France. The second CT scan was performed immediately after the first one, without re-positioning the patient. The Pneumo Chest Bellow (Lafayette Instrument, Lafayette, IN) was used to acquire the respiratory signal for the retrospective binning of CT scans in ten phase volumes $\left(\right.$ voxel size $\left.=1.17 \times 1.17 \times 3 \mathrm{~mm}^{3}\right)$. Patients P5-P7 were scanned with the GE LightSpeed RT16 CT scanner (GE Healthcare, Waukesha, WI) at Fondazione Salvatore Maugeri in Pavia, Italy. The respiratory signal was obtained from the real-time position management (RPM) system (Varian Medical Systems, Palo Alto, CA) placed on the patient's upper abdomen, with a resolution of $1.27 \times 1.27 \times 3.75 \mathrm{~mm}^{3}$ for the ten reconstructed volumes.

According to the adopted experimental protocol, the first acquired 4D CT (training 4D CT) was considered as the planning scan for the construction of the patient-specific respiratory motion model. The second 4D CT (testing 4D CT) was used to simulate the patient's anatomy at the time of irradiation for the assessment of the motion tracking accuracy, which was therefore based on a single average breathing cycle. For patients P5-P7, the surface surrogates used to drive the motion model were estimated from the RPM respiratory signal both for the training and for the testing 4D CTs. A single breathing cycle was obtained through a phasebased average of the different breathing cycles acquired during the CT scan. For patients $\mathrm{P} 1-\mathrm{P} 4$, the surface mesh corresponding to the thorax and upper abdomen was extracted from each training and testing 4D CT frame through threshold-based segmentation and Delaunay triangulation (figure 2). In order to obtain the spatial correspondence of surface points, the 4D CT surface meshes were non-rigidly registered (Schaerer et al 2012) to the reference mesh extracted from the training MidP CT image. For both 4D CTs, a mono-dimensional motion signal was derived for each surface point from the registered 3D coordinates by computing the Euclidean distance with respect to the most posterior position in the corresponding 4D CT scan. A single respiratory surrogate was synthesized for each 4D CT by averaging the motion signals of all surface points.

The proposed testing protocol consisted in three different experiments, as illustrated in figure 3. 
- The modeling test evaluated the inaccuracy associated with DIR procedures for model construction and with B-spline interpolation for the estimation of model output DVFs. The respiratory motion model was driven with the nominal parameters (constant unitary amplitude and 4D CT phase values: $0,10, \ldots 90 \%$ ) used to build the motion model. The estimated volumes were compared with the original frames of the training 4D CT.

- The rigid alignment test provided the uncertainty related to the standard clinical procedure for setup error correction, based on the rigid alignment of the patient's anatomy without any organ motion compensation. The global alignment to the nominal treatment position was simulated by rigidly registering the training MidP CT to the testing MidP $\mathrm{CT}$. The obtained roto-translation parameters were applied to each frame of the training 4D CT. The resulting aligned volumes were compared with the corresponding frames of the testing 4D CT.

- The tracking test quantified the effectiveness of the proposed approach in compensating for interfraction and intrafraction organ motion. The baseline shift between the two 4D CT scans was obtained by non-rigidly registering (Shackleford et al 2010) the training MidP CT to the testing MidP CT. The amplitude and phase parameters were calculated from the Hilbert transform of the surface surrogates extracted from the two 4D CT scans (figure 2). The surrogates, representing the single 4D CT breathing cycle, were replicated to form the cyclical function required for the computation of the Hilbert magnitude and phase angle information. For each testing 4D CT frame, the phase parameter was obtained as the interpolated position between the training frames that corresponds to the same Hilbert phase angle of the testing frame. The amplitude scaling factor was computed as the Hilbert magnitude of the testing frame divided by the magnitude of the training frame interpolated at the corresponding phase angle. The estimated output volumes were compared with the reference frames of the testing 4D CT.

For each described experimental test, the comparison between the estimated and reference 4D CT frames was based on the following variables (figure 3).

- The volume difference assessed the overall similarity between the estimated and reference volumes. The patient's body region was masked in each frame of the reference and estimated 4D CT scans. The absolute difference of voxel intensity in Hounsfield Unit (HU) was computed between the masked reference and estimated frames. The Pearson linear correlation between the reference and estimated HU values was also measured.

- The geometric errors evaluated the accuracy in reconstructing the position, shape and contours of the tumor and surrounding OARs (lungs, trachea and esophagus). The gross tumor volume (GTV) was contoured by a clinician on the maximum intensity projection (MIP) CT obtained from the first 4D CT scan, while OARs contours were delineated on the average CT scan. The clinical contours of all structures of interest were automatically propagated through DIR (Shackleford et al 2010) to each frame of the estimated and reference 4D CT scans (Orban de Xivry et al 2007). Geometric errors were quantified by measuring: (a) the 3D distance between the center of mass (COM) of the estimated and reference structures; (b) the Hausdorff distance (Huttenlocher et al 1993) between the point sets defining the estimated and reference structure contours; (c) the Dice coefficient between the estimated and reference structure volumes.

- The WEL variation $(\triangle W E L)$ quantified particle range fluctuations associated with the inaccuracy of the developed tracking method in estimating tissue density along the beam. Two particle beams were simulated from the ipsi-lateral and antero-posterior directions, as depicted in figure 4. Only for patient P7, which had a posterior tumor in the left lower lobe, the second beam was simulated in the posterior-anterior direction to avoid heart 


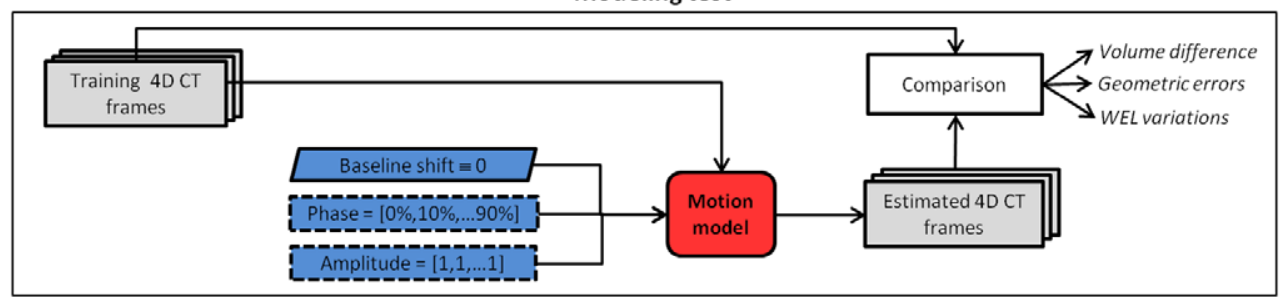

Rigid alignment test

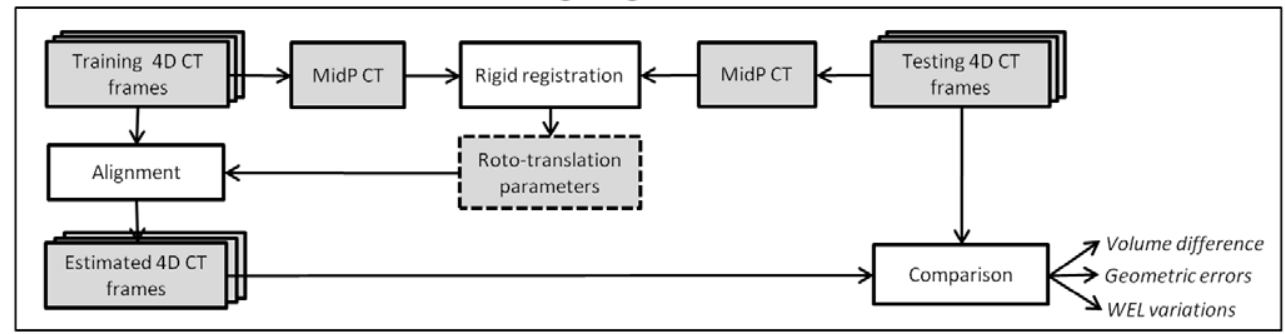

Tracking test

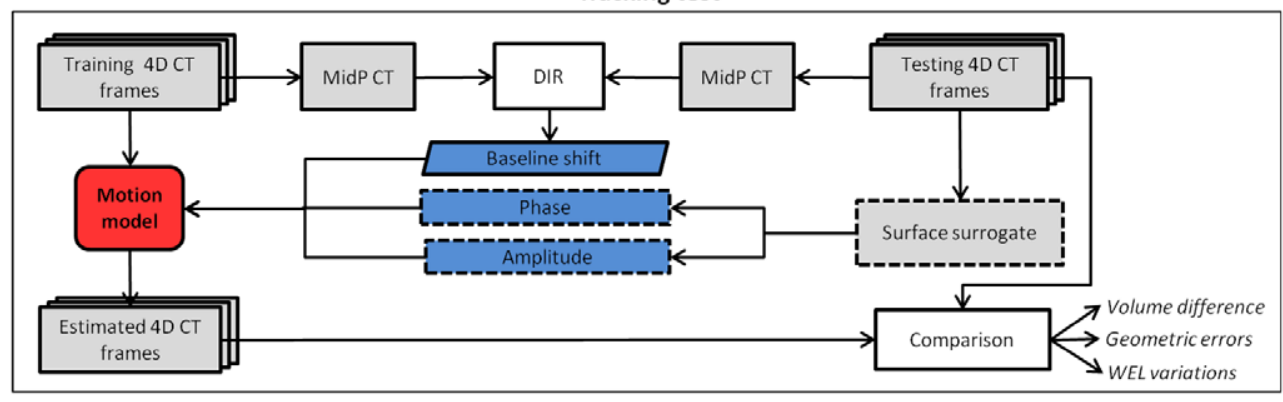

Legend: $\square$ =functional block; $\quad[-\mathbf{J}$ =1D signal; $\square$ =3D volumes (4D if stacked); $\square=3 \mathrm{D}$ DVF (4D if stacked).

Figure 3. Schematic representation of the proposed testing protocol, illustrating the procedural steps of the three performed experiments. The breathing parameters of the motion model are outlined in blue.

irradiation. The transversal section of the beams was chosen as the GTV extension in the corresponding direction, without applying any lateral margin. HU values of the estimated and reference 4D CT frames were converted into relative WEL values using the empirical relation proposed by Jäkel et al (2001). The relative WEL contribution of each voxel along the beam was integrated to obtain a WEL map within the tumor volume (figure 4). $\triangle$ WEL associated with a specific tumor voxel was calculated by subtracting the integrated WEL value of the estimated 4D CT frame from that of the corresponding reference frame.

The three described variables were computed for each corresponding frame of the reference and estimated 4D CT scans and the obtained results were then mediated over all frames. Due to the non-gaussian distribution of the measured variables, a non-parametric representation based on median value and inter-quartile range (IQR) was used to report the results.

The computational cost associated with the proposed tracking algorithm was quantified on a general-purpose laptop (2.2 GHz quad-core processor, $16 \mathrm{~GB}$ RAM). The temporal performance was evaluated by considering a VOI that includes the GTV and the beam entry channel, 

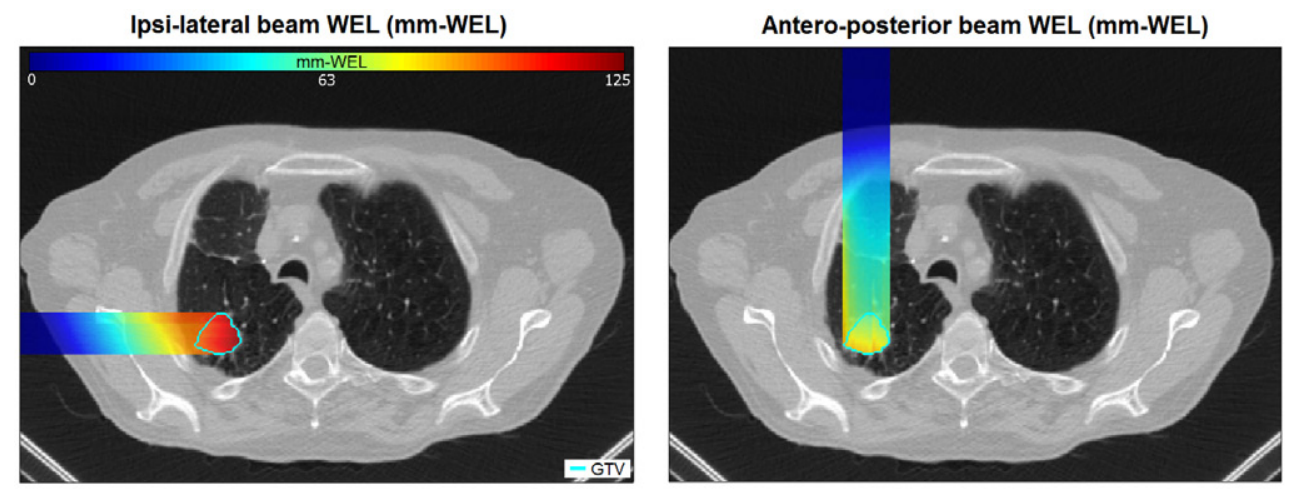

Figure 4. Axial slice from the end-exhale CT volume of patient P2. The tumor is contoured in cyan and the integrated WEL is superimposed along the simulated ipsilateral (left panel) and antero-posterior (right panel) beam lines.

with a margin of $20 \mathrm{~mm}$ in each spatial direction. In fact, a clinical application of the tracking method in charged particle therapy does not require the reconstruction of the entire CT volume for $\triangle \mathrm{WEL}$ estimation and range adaptation, but the motion model can be limited to the patient's anatomical region traversed by the beam.

\section{Results}

Table 2 reports for each patient an estimate of the baseline shifts measured between the training and testing 4D CT scans. This estimate was obtained by computing the 3D distance between the centroids of the different structures of interest (GTV and OARs) segmented in the corresponding 4D CT frames of the two scans. For patients P1-P3, who repeated the 4D CTs in the same day, baseline shifts were limited to $3.6 \mathrm{~mm}$. Patients P4-P7, who repeated the 4D CTs in different days, showed baseline shifts higher than $4.9 \mathrm{~mm}$, with a maximum value of $25.9 \mathrm{~mm}$ for patient P5. Table 2 also lists the amplitude scaling factor obtained for each patient between the two scans and the phase shift computed as the difference between the phase values estimated for each frame of the testing 4D CT and the corresponding nominal values (see section 2.2). The measured phase shifts did not exceed the $6.3 \%$ of the 4D CT frame interval. The amplitude scaling factor ranged from 0.3 (patient P5) to 1.9 (patient P2), meaning respectively a decrease of $70 \%$ and an increase of $90 \%$ in breathing motion magnitude between the two 4D CT scans.

Results concerning the volume difference for the three experimental tests are shown in figure 5, representing for each patient the absolute HU difference and Pearson correlation coefficient $(\rho)$ between the estimated and the corresponding reference volumes, mediated over all 4D CT frames. As shown in figure 5, the DIR and interpolation procedures for model construction (modeling test) introduced limited uncertainties in the estimated 4D CT frames, with volume differences lower than $18 \mathrm{HU}$ and $\rho>0.99$ for each patient. Relevant errors were measured when rigid registration was performed (rigid alignment test), with volume differences up to $60 \mathrm{HU}$ and $\rho=0.64$ (patient P7). The results were significantly improved (Wilcoxon signed-rank test, $p$-value $<10^{-12}$ ) after applying the proposed tracking approach (tracking test), obtaining $\rho>0.95$ in all patients. The median value $( \pm \mathrm{IQR})$ of volume differences over all patients was reduced from $39 \pm 17 \mathrm{HU}$ in the rigid alignment test to $22 \pm 3 \mathrm{HU}$ in the tracking test. 
Table 2. Baseline shift, amplitude scaling factor and phase shift measured between the two 4D CT scans for each patient, expressed as median \pm IQR values over all 4D CT frames.

\begin{tabular}{|c|c|c|c|c|c|c|}
\hline \multirow[b]{2}{*}{ Patient } & \multicolumn{4}{|c|}{ Baseline shift (mm) } & \multirow{2}{*}{$\begin{array}{l}\text { Amplitude } \\
\text { scaling factor }\end{array}$} & \multirow{2}{*}{$\begin{array}{l}\text { Phase shift } \\
(\%)\end{array}$} \\
\hline & Tumor & Lungs & Trachea & Esophagus & & \\
\hline$\overline{\mathrm{P} 1}$ & $3.6 \pm 0.7$ & $2.8 \pm 0.2$ & $1.7 \pm 0.7$ & $1.6 \pm 0.6$ & $0.9 \pm 0.05$ & $1.8 \pm 1.5$ \\
\hline $\mathrm{P} 2$ & $0.7 \pm 0.5$ & $2.0 \pm 1.8$ & $3.1 \pm 0.8$ & $2.1 \pm 1.5$ & $1.9 \pm 0.2$ & $2.0 \pm 2.1$ \\
\hline P3 & $2.8 \pm 0.7$ & $2.1 \pm 0.7$ & $0.9 \pm 0.3$ & $1.1 \pm 0.4$ & $1.2 \pm 0.09$ & $6.3 \pm 3.1$ \\
\hline P4 & $4.9 \pm 0.9$ & $5.5 \pm 1.1$ & $6.6 \pm 2.0$ & $7.6 \pm 0.9$ & $0.9 \pm 0.06$ & $0.6 \pm 0.9$ \\
\hline P5 & $25.9 \pm 1.1$ & $22.1 \pm 3.0$ & $21.8 \pm 1.6$ & $18.6 \pm 1.9$ & $0.3 \pm 0.01$ & $0.7 \pm 0.5$ \\
\hline P6 & $5.6 \pm 1.1$ & $9.9 \pm 1.0$ & $5.8 \pm 1.7$ & $9.1 \pm 1.3$ & $0.8 \pm 0.04$ & $1.4 \pm 1.2$ \\
\hline P7 & $15.3 \pm 0.4$ & $17.4 \pm 0.6$ & $21.3 \pm 1.8$ & $17.0 \pm 0.4$ & $0.9 \pm 0.01$ & $0.1 \pm 0.1$ \\
\hline
\end{tabular}
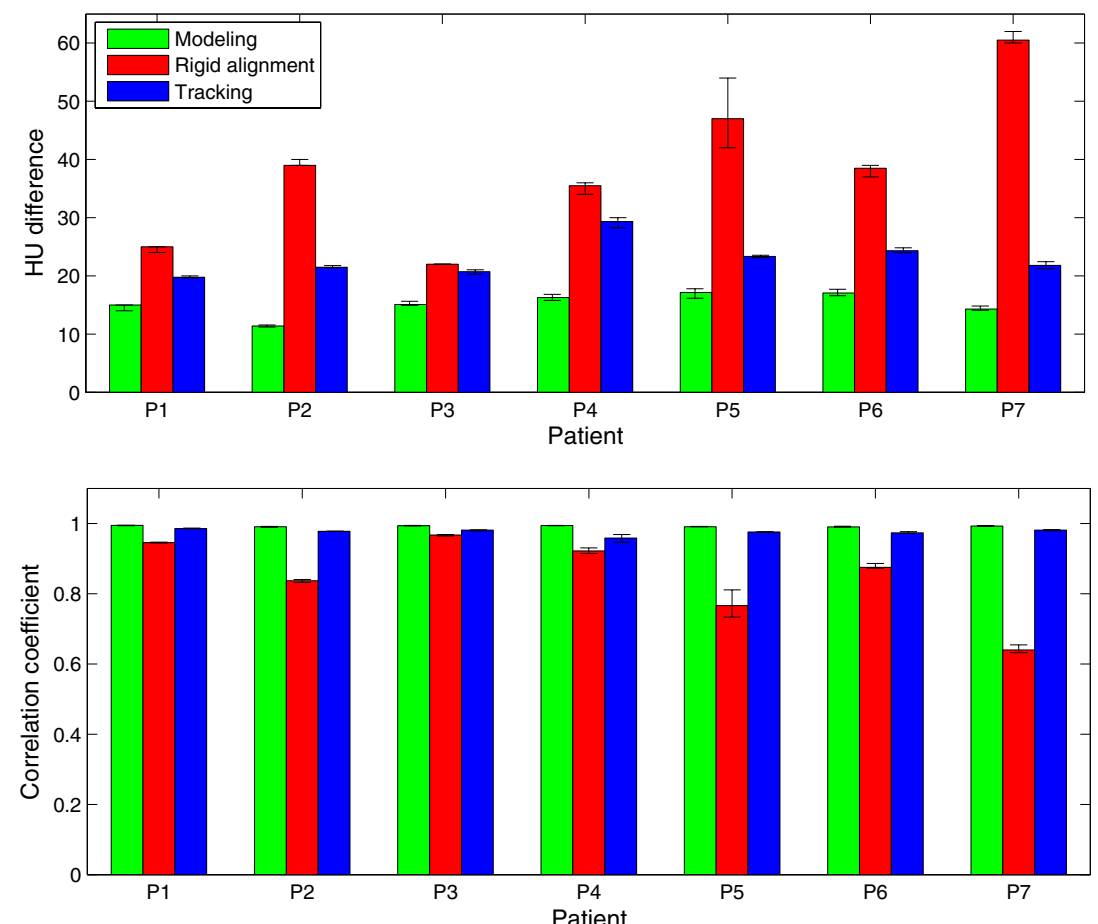

Figure 5. Absolute difference (upper panel) and Pearson correlation coefficient (lower panel) between HU voxel values of corresponding reference and estimated 4D CT frames (median $\pm \mathrm{IQR}$ ) for the modeling, rigid alignment and tracking tests.

Figure 6 depicts the geometric errors in reconstructing the position, shape and volume of the GTV and surrounding OARs, mediated over all 4D CT frames. In the tracking test, tumor COM distances between the estimated and reference CT frames ranged between 0.6 and 1.7 $\mathrm{mm}$ across all patients. The overall median error in position reconstruction was lower than $1.3 \mathrm{~mm}$ for the surrounding OARs, as reported in table 3. Errors in shape recon-struction, quantified by the Hausdorff distance, did not exceed $1.3 \mathrm{~mm}$ for all structures of interest (figure 6), with median values lower than $0.7 \mathrm{~mm}$ over all patients (table 3). The Dice coefficient for volume overlap was on average 0.8 and 0.9 for the GTV and OARs, 

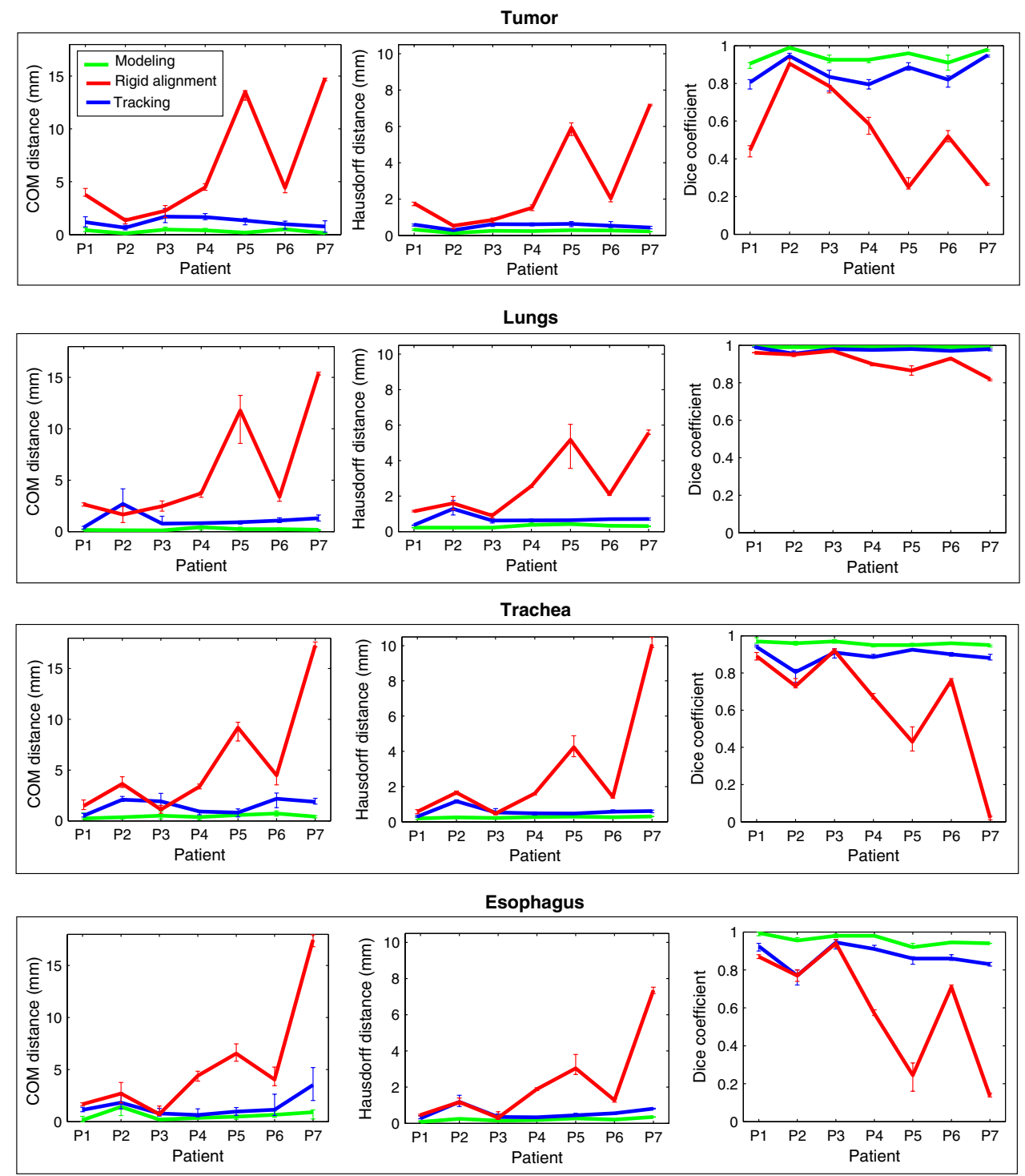

Figure 6. Geometric errors in reconstructing the position (COM distance), shape (Hausdorff distance) and volume (Dice coefficient) of tumor, lungs, trachea and esophagus for the different experiments. The figure reports for each patient the median value \pm IQR computed over all frames of the estimated 4D CT.

respectively. The accuracy of the proposed tracking approach was significantly $\mathrm{h}$ igher (Wilcoxon signed-rank test, $p$-value $<10^{-4}$ ) with respect to the rigid alignment test, show-ing tumor COM distances up to $14.8 \mathrm{~mm}$ and Dice coefficients lower than 0.3 (figure 6). COM and Hausdorff distances for all structures of interest were reduced on average by $70 \%$ and $67 \%$, respectively, in the tracking test. We did not observe any relevant geometric uncertainty associated with the DIR-based modeling and interpolation processes (modeling test), obtaining tumor COM and Hausdorff distances lower than 0.5 $\mathrm{mm}$ and Dice coeffi-cient higher than 0.9 for each patient (figure 6). 
Table 3. Accuracy of the proposed tracking approach in reconstructing the tumor and surrounding OARs, expressed as median \pm IQR of the different geometric variables measured over all 4D CT frames and all patients.

\begin{tabular}{llll}
\hline Structure of interest & $\begin{array}{l}\text { COM } \\
\text { distance }(\mathrm{mm})\end{array}$ & $\begin{array}{l}\text { Hausdorff } \\
\text { distance }(\mathrm{mm})\end{array}$ & Dice coefficient \\
\hline Tumor & $1.16 \pm 0.91$ & $0.52 \pm 0.23$ & $0.84 \pm 0.13$ \\
Lungs & $0.89 \pm 0.64$ & $0.66 \pm 0.19$ & $0.98 \pm 0.01$ \\
Trachea & $1.29 \pm 1.46$ & $0.51 \pm 0.28$ & $0.90 \pm 0.04$ \\
Esophagus & $1.23 \pm 1.38$ & $0.50 \pm 0.45$ & $0.88 \pm 0.08$ \\
\hline
\end{tabular}

Table 4. Absolute WEL variation measured within the tumor volume for the ipsi-lateral and antero-posterior simulated beams in the different experimental tests. The median \pm IQR values computed over the ten 4D CT frames are reported for each patient.

\begin{tabular}{lllllll}
\hline & \multicolumn{5}{c}{ Absolute $\Delta$ WEL (mm-WEL) } \\
\cline { 2 - 7 } & \multicolumn{7}{c}{ Ipsi-lateral beam } & \multicolumn{3}{c}{ Antero-posterior beam } \\
\cline { 2 - 7 } Patient & Modeling & Tracking & Rigid & Modeling & Tracking & Rigid \\
\hline P1 & $0.42 \pm 0.06$ & $1.00 \pm 0.23$ & $1.73 \pm 0.15$ & $0.61 \pm 0.24$ & $0.95 \pm 0.60$ & $1.04 \pm 0.25$ \\
P2 & $0.30 \pm 0.05$ & $0.67 \pm 0.10$ & $1.67 \pm 0.39$ & $0.65 \pm 0.22$ & $1.30 \pm 0.53$ & $2.71 \pm 0.32$ \\
P3 & $0.49 \pm 0.15$ & $0.72 \pm 0.22$ & $2.33 \pm 0.10$ & $0.72 \pm 0.28$ & $1.03 \pm 0.60$ & $2.65 \pm 0.39$ \\
P4 & $0.56 \pm 0.42$ & $1.36 \pm 0.92$ & $7.95 \pm 0.56$ & $0.74 \pm 0.53$ & $1.40 \pm 0.32$ & $4.49 \pm 0.43$ \\
P5 & $0.38 \pm 0.10$ & $0.85 \pm 0.16$ & $4.31 \pm 0.42$ & $0.81 \pm 0.39$ & $1.64 \pm 1.03$ & $3.90 \pm 0.63$ \\
P6 & $0.70 \pm 0.67$ & $1.55 \pm 0.72$ & $1.86 \pm 0.64$ & $0.80 \pm 0.80$ & $1.89 \pm 0.83$ & $2.43 \pm 0.59$ \\
P7 & $0.37 \pm 0.03$ & $0.79 \pm 0.14$ & $3.90 \pm 0.11$ & $0.31 \pm 0.06$ & $0.48 \pm 0.13$ & $9.39 \pm 0.42$ \\
Median & $0.41 \pm 0.20$ & $0.90 \pm 0.43$ & $2.33 \pm 2.21$ & $0.70 \pm 0.45$ & $1.33 \pm 0.81$ & $3.03 \pm 1.94$ \\
\pm IQR & & & & & & \\
\hline
\end{tabular}

Table 4 lists the median value of absolute WEL variation within the tumor volume obtained with the ipsi-lateral and antero-posterior simulated beams. In the modeling test, $\triangle \mathrm{WEL}$ mediated over all 4D CT frames was limited to $0.8 \mathrm{~mm}$-WEL for each patient. WEL difference ranged between 1.0 and $9.4 \mathrm{~mm}$-WEL for the rigid alignment test. A significant improvement (Wilcoxon signed-rank test, $p$-value $<10^{-10}$ ) was obtained by applying the proposed tracking method (tracking test), showing an overall median $\triangle \mathrm{WEL}$ of $0.9 \mathrm{~mm}$ and $1.3 \mathrm{~mm}$-WEL for the ipsi-lateral and antero-posterior beams, respectively (table 4). We did not find any statistically significant correlation (Pearson coefficient $\rho=0.6, p$-value $>0.1$ ) between WEL errors and tumor range of motion (table 1). The signed distributions of $\triangle \mathrm{WEL}$ measured for each tumor voxel with the ipsi-lateral beam are depicted in figure 7 . In the tracking test, $\Delta \mathrm{WEL}$ distribution was limited to $\pm 6 \mathrm{~mm}$-WEL in all patients, whereas $\triangle \mathrm{WEL}$ for the rigid alignment test reached up to $-28 \mathrm{~mm}-\mathrm{WEL}$.

Table 5 lists for each patient the computational time associated to the DVF interpolation and warping procedures required for the reconstruction of the selected VOI including the tumor and the beam entry channel, mediated over all 4D CT frames. Results were lower than $0.75 \mathrm{~s}$ for each patient, with median values of $0.42 \mathrm{~s}$ and $0.48 \mathrm{~s}$ for the ipsilateral and antero-posterior beam directions, respectively. A linear correlation (Pearson coefficient $\rho=0.96, p$-value $<10^{-7}$ ) was found between the computational cost and the volume size. 

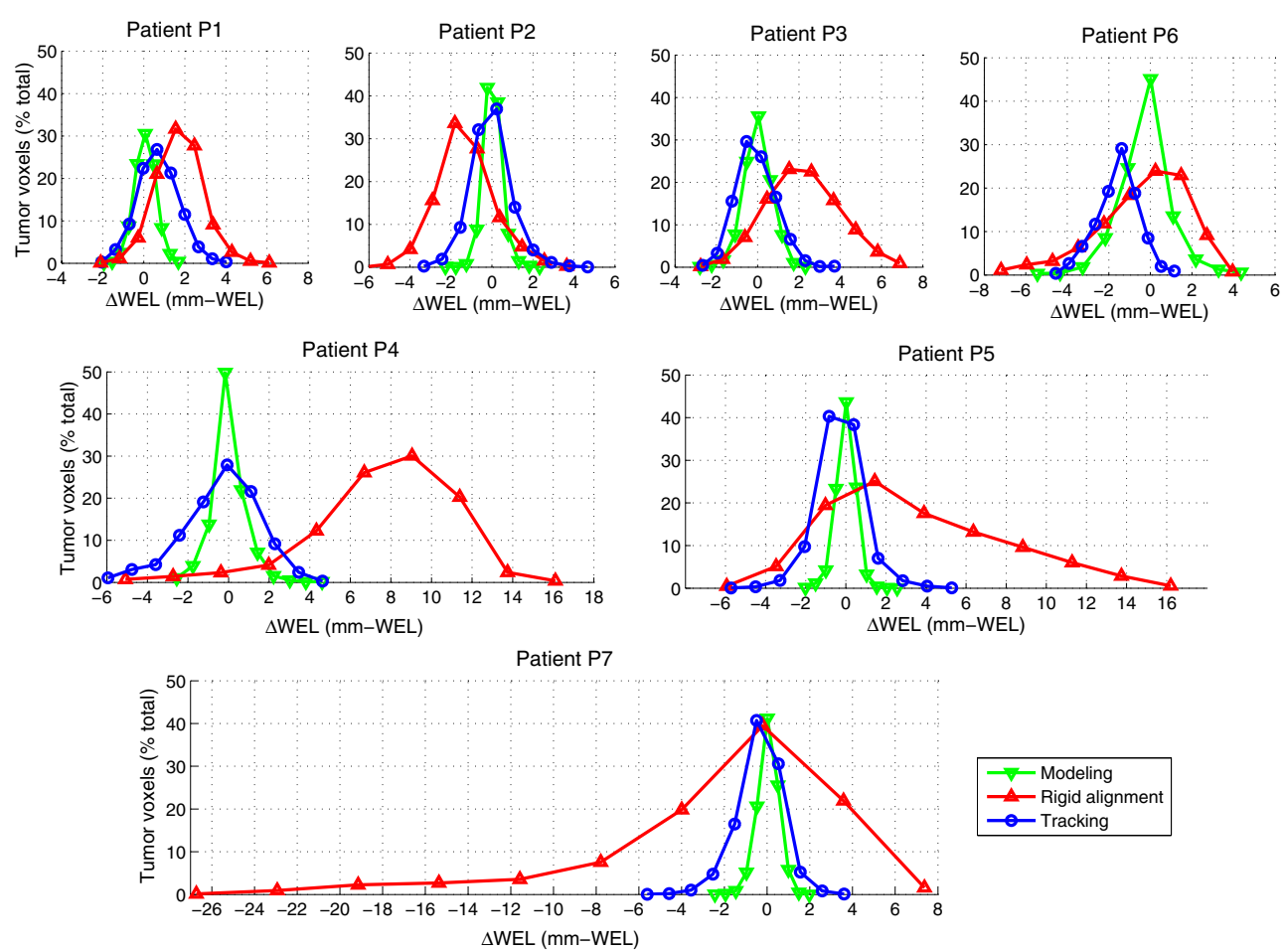

Figure 7. Signed distributions of $\triangle \mathrm{WEL}$ obtained for each tumor voxel with the particle beam simulated in the ipsi-lateral direction in the different experiments.

Table 5. Computational time required for the reconstruction of the VOI selected for the ipsi-lateral and antero-posterior simulated beams. The median $\pm \mathrm{IQR}$ values computed over the ten 4D CT frames are reported for each patient.

\begin{tabular}{lll}
\hline & \multicolumn{2}{c}{ Computational time (s) } \\
\cline { 2 - 3 } Patient & Ipsi-lateral beam & Antero-posterior beam \\
\hline P1 & $0.46 \pm 0.03$ & $0.48 \pm 0.03$ \\
P2 & $0.64 \pm 0.05$ & $0.75 \pm 0.05$ \\
P3 & $0.39 \pm 0.02$ & $0.60 \pm 0.04$ \\
P4 & $0.38 \pm 0.02$ & $0.34 \pm 0.02$ \\
P5 & $0.40 \pm 0.04$ & $0.46 \pm 0.04$ \\
P6 & $0.17 \pm 0.01$ & $0.15 \pm 0.02$ \\
P7 & $0.56 \pm 0.04$ & $0.48 \pm 0.02$ \\
Median \pm IQR & $0.42 \pm 0.18$ & $0.48 \pm 0.22$ \\
\hline
\end{tabular}

\section{Discussion}

A tumor tracking method based on a surrogate-driven 4D CT motion model is proposed for organ motion compensation in extracranial radiation treatments with charged particle therapy. The application of tracking techniques in particle treatments is challenging due to the need to compensate both for target motion and for radiological path length variations in the tissues traversed by the beam. Current tracking methods applied in conventional radiotherapy (Kilby 
et al 2010, Keall et al 2014) are based on the dynamic localization of single internal points, which is not sufficient to estimate particle range uncertainties associated with density varia-tions along the beam. Conversely, the proposed motion modeling approach provides the daily dynamics due to respiration of the entire patient's anatomy scanned in the planning CT. This allows obtaining complete density and WEL information required for particle beam range adaptation.

The developed method provides several benefits compared to surrogate-driven motion models previously proposed in the literature (McClelland et al 2011, Fayad et al 2012, Martin et al 2013). Differently from the rigid translational approach applied by Martin et al (2013), we used a deformable motion model that accounts for non-rigid deformations of patient's internal anatomy. The accuracy and robustness of our modeling approach was further improved by choosing as reference frame the MidP CT image that contains reduced noise and artefacts with respect to the individual 4D CT frames (Wolthaus et al 2008), e.g. the end-exhale volume used by McClelland et al (2011) and Fayad et al (2012). Moreover, we combined three different breathing parameters to build and update the motion model, which proved to be substantially more robust than considering a single parameter (Fayad et al 2012, Fassi et al 2014). We also introduced specific strategies to compensate for the day-today variations in motion param-eters, which were not taken into account in the previous modeling methods (Fayad et al 2012, Martin et al 2013). In this study, the baseline shifts were corrected using a DIR approach, which was shown to significantly improve the tracking accuracy in different lung regions with respect to the rigid alignment procedure, as performed by McClelland et al (2011). The amplitude changes were compensated though a scaling factor obtained by comparing surface motion during planning and treatment. The phase parameter was estimated using the Hilbert transform approach, which allows capturing the hysteresis and irregular dynamics in respira-tory motion, unlike the linear interpolation method applied in McClelland et al (2011).

Differently from our previous works (Vandemeulebroucke et al 2009, Fassi et al 2014), in the present study the proposed tracking method was specifically developed and tested for a possible application in charged particle therapy, by using a dataset of double 4D CTs acquired from seven lung cancer patients. The method performance was evaluated both from a geometrical point of view, by assessing the accuracy in localizing not only the tumor but also surrounding OARs, and from a radiological point of view, by quantifying the associ-ated WEL variation that influence particle beam range. The delineations of the structures of interest used for model evaluation were obtained through DIR-based contour propagation, which was proven to be comparable to the inter-observer variability (Orban de Xivry et al 2007). Although the DIR algorithm for model construction and contour propagation was the same (Shackleford et al 2010), the obtained DVFs were independent since different reference images were employed, i.e. the MidP CT for model construction and the MIP/average CT for contour propagation.

Concerning the geometrical accuracy, the median localization errors for the target and surrounding OARs obtained with the proposed tracking method were lower than $1.3 \mathrm{~mm}$, with Dice coefficients for volume overlap higher than 0.8 (table 3). The uncertainties associated with the DIR procedures for model construction and contour propagation, assessed with the modeling test, were limited to $0.5 \mathrm{~mm}$ (figure 6), which is only one third of the total tracking error, thus demonstrating the high reliability of the applied modeling and testing strategies. The obtained results are very promising with respect to those published for previous motion modeling methods (McClelland et al 2011, Fayad et al 2012, Martin et al 2013), likely due to the introduced optimization strategies accounting for interfraction and intrafraction respira-tory motion variations. The performance achieved with the proposed tracking technique was significantly superior compared with the standard rigid alignment approach, thus proving the 
importance of the applied DIR-based techniques for organ motion compensation. As depicted in figure 6, the interfraction variations measured for patients P4-P7, which repeated the 4D CTs in different days, were not adequately corrected with the rigid procedure, showing residual localization errors higher than $15 \mathrm{~mm}$ and quasi-null volume overlap coefficients. Conversely, the proposed tracking method was able to fully compensate both for the considerable baseline shifts measured for patients P5, P7 and for the large amplitude changes obtained for patients P2, P5 (table 2). Patient P1 proved the ability of the tracking technique in compensating also for intrafraction variations in the breathing parameters, due for example to a possible patient relaxation between the two $4 \mathrm{D}$ CTs, resulting in considerable tumor COM errors with the rigid approach (figure 7).

Radiological WEL variation within the target volume ranged in $\pm 6 \mathrm{~mm}$-WEL with the developed tracking technique (figure 7), with median absolute values lower than $1.9 \mathrm{~mm}$ WEL for all patients (table 4). The measured $\triangle \mathrm{WEL}$ was symmetrically distributed with zero median, thus implying an accurate longitudinal dose deposition. Beam undershoot or over-shoot to the target can be instead expected with the rigid alignment procedure, due to the associated relevant shifts in $\triangle \mathrm{WEL}$ distribution (figure 7). The results obtained with the proposed tracking method are comparable to the performance of the respiratory-gated method tested by Kumagai et al (2009) for charged particle pancreatic therapy, showing WEL variation from -1.5 to $1.3 \mathrm{~mm}$ in case of regular breathing and from -9.1 to $8.1 \mathrm{~mm}$ for irregular breathing. However, in that study (Kumagai et al 2009) $\triangle$ WEL was evaluated only for the end-exhale phase that is more stable and reproducible, while in our work all breathing phases were considered. Moreover, the abdominal region analysed for pancreatic cancers (Kumagai et al 2009) is mostly uniform in density, differently from the thoracic region assessed in our case for lung lesions.

The accuracy of the investigated motion model can be affected by artifacts in 4D CT volumes, mainly induced by breathing irregularities or incorrect phase binning. For example, the shift in $\triangle \mathrm{WEL}$ distribution from zero median measured for patient P6 (figure 7) can be likely attributed to the presence of respiratory motion artifacts in the abdominal region, especially for the inspiratory CT frames. In this study, an abdominal belt or the RPM system were used for 4D CT image sorting, but more robust approaches, based for example on surface imaging (Spadea et al 2011) or multiple point methods (Gianoli et al 2011), might be employed for improving breathing phase identification and reducing image artifacts. A multi B-spline transform method could also be applied to take into account the lung sliding motion in deformable registration of thoracic CT images (Delmon et al 2013). The localized approach based on VOI selection proved effective in speeding up DVF procedures for volume reconstruction, obtaining a temporal performance better than $0.75 \mathrm{~s}$ for all patients (table 5). Although a further computational optimization of the tracking algorithm is required, the reported results can be considered promising in view of full real-time implementation.

The application of the developed tracking method in a clinical scenario involves some differences with respect to the present experimental testing on 4D CT datasets, since the input parameters of the respiratory motion model will be retrieved and updated at each treatment session based on in-room x-ray imaging and optical surface localization (Fassi et al 2014). In this work, two noise-free MidP CT volumes were non-rigidly registered for baseline shift cor-rection, while in the actual tumor tracking application DIR procedures will likely involve the CBCT volume acquired during patient setup (Fassi et al 2014), which is affected by blurring due to breathing motion. The use of a motion-compensated CBCT with image-blur reduction (Rit et al 2009) is expected to limit DIR errors for baseline update. In addition, in the present study the external respiratory surrogate was extracted by using the RPM signal, derived from a single abdominal control point, or by segmenting the patient's thoracic surface from the 
4D CT volumes, potentially affected by artifacts. In the actual application, optical surface imaging systems with sub-millimetric accuracy (Bert et al 2005) will likely be applied for the reconstruction of the entire thoraco-abdominal surface (Fassi et al 2014), thus increasing the robustness in the external surrogate estimation. The estimation of amplitude and phase param-eters with the Hilbert transform for the real-time feeding to the motion model will require the inclusion of breathing signal history to form the cyclical function. A single previous respira-tory cycle can be considered, as performed in the present study, in order to limit the delay in sensing breathing irregularities. Finally, an inroom intrafraction assessment of the motion model reliability, based for example on x-ray projection acquisition, is envisaged for correla-tion verification and update over the entire treatment course.

\section{Conclusions}

The present work can be regarded as a feasibility study for the potential extension of tumor tracking techniques to the treatment of intrafraction moving lesions with charged particle therapy. This will allow exploiting the excellent geometrical accuracy and dose conformity of scanned beams for improving local tumor control and normal tissue sparing in extracra-nial treatments. The clinical applicability of the proposed tracking approach will necessarily require the experimental testing on a wider number of patients, which can be for example realized by considering 4D CBCT data (Rit et al 2009) acquired in different treatment sessions.

\section{Acknowledgments}

The authors would like to thank $\mathrm{J}$ Vandemeulebroucke and $\mathrm{M}$ Fernandes from Centre Léon Bérard, Lyon, France for contributing to the design and implementation of the deformable breathing motion model.

\section{References}

Balter J M et al 2005 Accuracy of a wireless localization system for radiotherapy Int. J. Radiat. Oncol. Biol. Phys. 61 933-37

Baroni G, Riboldi M, Spadea M F, Tagaste B, Garibalidi C, Orecchia R and Pedotti A 2007 Integration of enhanced optical tracking techniques and imaging in IGRT J. Radiat. Res. 48 A61-74

Bert C and Durante M 2011 Motion in radiotherapy: particle therapy Phys. Med. Biol. 56 R113-44

Bert C, Metheany K G, Doppke K and Chen G T 2005 A phantom evaluation of a stereo-vision surface imaging system for radiotherapy patient setup Med. Phys. 32 2753-62

Delmon V, Rit S, Pinho R and Sarrut D 2013 Registration of sliding objects using direction dependent B-splines decomposition Phys. Med. Biol. 58 1303-14

Depuydt T et al 2013 Initial assessment of tumor tracking with a gimbaled linac system in clinical circumstances: a patient simulation study Radiother. Oncol. $106236-40$

Fassi A, Schaerer J, Fernandes M, Riboldi M, Sarrut D and Baroni G 2014 Tumor tracking method based

on a deformable 4D CT breathing motion model driven by an external surface surrogate Int.

J. Radiat. Oncol. Biol. Phys. 88 182-8

Fayad H, Pan T, Pradier O and Visvikis D 2012 Patient specific respiratory motion modeling using a

3D A multiple points method for 4D CT image sorting Med. Phys. 38 656-67 patient's external surface Med. Phys. 39 3386-95

Gabor D 1946 Theory of communications J. Inst. Elect. Eng. 93 429-57

Gianoli C, Riboldi M, Spadea M F, Travaini L L, Ferrari M, Mei R, Orecchia R and Baroni G 2011 
Hoogeman M J, Prevost B, Nuyttens J, Poll J, Levendag P and Heumen B 2009 Clinical accuracy of the respiratory tumor tracking system of the Cyberknife: assessment by analysis of log files Int.

J. Radiat. Oncol. Biol. Phys. 74 297-303

Huttenlocher D P, Klanderman G A and Rucklidge W G 1993 Comparing images using the Hausdorff distance IEEE Trans. Pattern Anal. Mach. Intell. 15 850-63

Imura $\mathrm{M}$ et al 2005 Insertion and fixation of fiducial markers for setup and tracking of lung tumors

in radiotherapy Int. J. Radiat. Oncol. Biol. Phys. 63 1442-7

Jäkel O, Jacob C, Schardt D, Karger C P and Hartmann G H 2001 Relation between carbon-ion ranges and x-ray CT numbers Med. Phys. 28 701-3

Keall P J, Colvill E, O’Brien R, Ng J A, Poulsen P R, Eade T, Kneebone A and Booth J T 2014 The first clinical implementation of electromagnetic transponder-guided MLC tracking Med. Phys. 41020702

Keall P J, Kini V R, Vedam S S and Mohan R 2001 Motion adaptive x-ray therapy: a feasibility study Phys. Med. Biol. 46 1-10

Keall P J et al 2006 The management of respiratory motion in radiation oncology: report of AAPM Task Group 76 Med. Phys. 33 3874-900

Kilby W, Dooley J R, Kuduvalli G, Sayeh S and Maurer C R Jr 2010 The Cyberknife robotic

radiosurgery system in 2010 Technol. Cancer Res. Treat. 9 433-52

Kumagai M, Mori S, Hara R, Asakura H, Kishimoto R, Kato H, Yamada S and Kandatsu S 2009

Water-equivalent pathlength reproducibility due to respiratory pattern variation in charged-

particle pancreatic radiotherapy Radiol. Phys. Technol. 2 112-8

Martin J, McClelland J R, Yip C, Thomas C, Hartill C, Ahmad S, O'Brien R, Meir I, Landau D and Hawkes D 2013 Building motion models of lung tumours from cone-beam CT for radiotherapy applications Phys. Med. Biol. 58 1809-22

McClelland J R, Hughes S, Modat M, Qureshi A, Ahmad S, Landau D B, Ourselin S and Hawkes D J 2011 Interfraction variations in respiratory motion models Phys. Med. Biol. 56 251-72

Mori S, Chen G T and Endo M 2007 Effects of intrafractional motion on water equivalent path length in respiratory gated heavy charged particle beam radiotherapy Int. J. Radiat. Oncol. Biol.

Phys. $69308-17$

Mori S, Wolfgang J, Lu H M, Schneider R, Choi N C and Chen G T Y 2008 Quantitative assessment of range fluctuations in charged particle lung irradiation Int. J. Radiat. Oncol. Biol. Phys. 70 253-61

Orban de Xivry J, Janssens G, Bosmans G, De Craene M, Dekker A, Buijsen J, van Baardwijk A, De Ruysscher D, Macq B and Lambin P 2007 Tumor delineation and cumulative dose computation in radiotherapy based on deformable registration of respiratory correlated CT images of lung cancer patients Radiother. Oncol. 85 232-8

Poulsen P R, Cho B, Sawant A, Ruan D and Keall P J 2010 Dynamic MLC tracking of moving targets with a single $\mathrm{kV}$ imager for 3D conformal and IMRT treatments Acta Oncol. 49 1092-100

Riboldi M, Orecchia R and Baroni G 2012 Real-time tumour tracking in particle therapy: technological developments and future perspectives Lancet Oncol. 13 e383-91

Rit S, Wolthaus J W, van Herk M and Sonke J J 2009 On-the-fly motion-compensated cone-beam CT using an a priori model of the respiratory motion Med. Phys. 36 2283-96

Saito N, Bert C, Chaudhri N, Gemmel A, Schardt D, Durante M and Rietzel E 2009 Speed and

accuracy of a beam tracking system for treatment of moving targets with scanned ion beams Phys.

Med. Biol. 54 4849-62

Schaerer J, Fassi A, Riboldi M, Cerveri P, Baroni G and Sarrut D 2012 Multi-dimensional respiratory motion tracking from markerless optical surface imaging based on deformable mesh registration Phys. Med. Biol. 57 357-73

Schardt D, Elsässer T and Schulz-Ertner D 2010 Heavy-ion tumor therapy: physical and radiobiological benefits Rev. Mod. Phys. 82 383-425

Schweikard A, Shiomi H and Adler J R 2004 Respiration tracking in radiosurgery Med. Phys. 31 2738-41

Seregni M, Cerveri P, Riboldi M, Pella A and Baroni G 2012 Robustness of external/internal correlation models for real-time tumor tracking to breathing motion variations Phys. Med. Biol. 57 7053-74

Shackleford J A, Kandasamy N and Sharp G C 2010 On developing B-spline registration algorithms for multi-core processors Phys. Med. Biol. 55 6329-51

Shirato H et al 2000 Physical aspects of a real-time tumor-tracking system for gated radiotherapy Int.

J. $\quad$ Radiat. Oncol. Biol. Phys. 48 1187-95 
Spadea M F, Baroni G, Gierga D P, Turcotte J C, Chen G T and Sharp G C 2011 Evaluation and commissioning of a surface based system for respiratory sensing in 4D CT J. Appl. Clin. Med.

Phys. 12 162-9

Takayama K et al 2009 Initial validations for pursuing irradiation using a gimbals tracking system Radiother. Oncol. 93 45-9

Vandemeulebroucke J, Kybic J, Clarysse P and Sarrut D 2009 Respiratory motion estimation from conebeam projections using a prior model Med. Image Comput. Comput. Assist. Interv. 12 365-72

Wolthaus J W, Sonke J J, van Herk M and Damen E M 2008 Reconstruction of a time-averaged midposition CT scan for radiotherapy planning of lung cancer patients using deformable registration Med. Phys. 35 3998-4011 